\title{
NON-DESTRUCTIVE EVALUATION FOR HORIZONTAL CRACKS IN EXISTING RC SLABS BY ANALYSIS-AIDED IMPACT ELASTIC-WAVE METHODS
}

\author{
Heung-Soo Lee \\ Graduate School of Engineering, Osaka University, 2-1 Yamadaoka, Suita, Osaka, Japan \\ lee_heungsoo@civil.eng.osaka-u.ac.jp \\ Toshiro Kamada \\ Graduate School of Engineering, Osaka University, 2-1 Yamadaoka, Suita, Osaka, Japan \\ kamada@civil.eng.osaka-u.ac.jp \\ Shinya Uchida \\ Graduate School of Science and Engineering, Saga University, 1 Honjo, Saga, Saga, Japan \\ uchida@cc.saga-u.ac.jp \\ Toshiki Iwasaki \\ Graduate School of Engineering, Osaka University, 2-1 Yamadaoka, Suita, Osaka, Japan \\ t.iwasaki@civil.eng.osaka-u.ac.jp
}

\begin{abstract}
In this study, impact elastic-wave method was performed at the bottom surface of RC slab cut from an existing highway bridges to survey the horizontal cracks. Before measurements by impact elastic-wave method, impact response analysis was applied to determine optimal steel ball diameter, distance between impact point and receiving point of elastic-wave. Efficiency of analysis-aided impact elastic-wave method was confirmed by drilling and observing the interior of the RC slab by stick scanner. Evaluation results by this method agreed well with results of visual inspection. Thus, validity of the analysis-aided impact elastic-wave method on detection of horizontal cracks in RC slabs of highway bridges was demonstrated.
\end{abstract}

Keywords: RC slabs; horizontal cracks; non-destructive testing; impact elastic-wave methods.

\section{Introduction}

Horizontal cracks have been recently found in cut cross-sections of deteriorated RC slabs of highway bridges in Japan (shown in Fig. 1) ${ }^{1}$.

In this study, impact elastic-wave method was performed at the bottom surface of an $\mathrm{RC}$ slab cut out from an existing highway bridge to survey horizontal cracks. Before measurements by impact elastic-wave method, impact response analysis was applied to determine elastic-wave input method and elastic-wave detection method. 


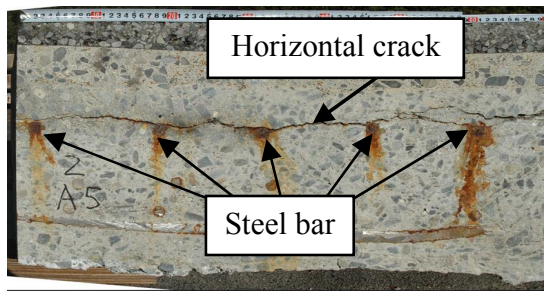

Fig. 1. Horizontal cracks in cut cross-sections of RC slab.

\section{Outline of a Reinforced Concrete Slab Cut from a Highway Bridge}

An outline and dimensions of a RC slab cut out from a highway bridge is shown in Fig. 2. The thickness of the RC slab was increased by additionally applying steel fiber reinforced concrete of a thickness of $60 \mathrm{~mm}$ and an asphalt pavement of the same thickness. The slab was approximately $2000 \mathrm{~mm}$ in width, $6400 \mathrm{~mm}$ in length and $320 \mathrm{~mm}$ in thickness.

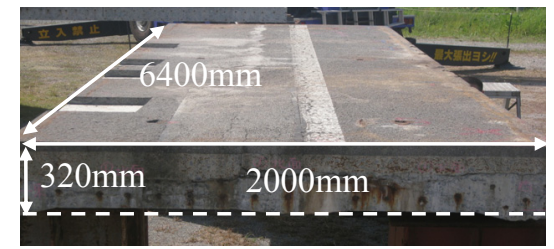

Fig. 2. Outline of a RC slab used in an existing highway bridge.

\section{Outline of the Proposed Method}

This chapter describes a method for determining an appropriate combination of "I: elastic-wave input method" and "II: elastic-wave detection method" for a RC slab cut from a highway bridge, through 3-dimensional impact elastic analysis. In general, it is not so easy to correctly simulate propagation behavior of elastic wave, especially ultrasonic wave (the wavelength is relatively small) in concrete because concrete is inhomogeneous material that contains aggregates and voids in cement paste matrix. However, when the impact elastic-wave method is applied, as elastic wave of relatively long wavelength is used, reflection of wave at interfaces between aggregates/voids and cement paste matrix would rarely occur. So, in the case of using the impact elastic-wave method, concrete can be thought as homogeneous material. Then we can utilize impact response analysis to simulate elastic behavior in target concrete members. That is why we apply this type of analysis for this study.

\subsection{Analysis model}

The width and the length of the analysis model were set at $2000 \mathrm{~mm}$ based on the results of past studies of the authors ${ }^{2}$ to minimize effects of reflection at side surfaces of the model. The thickness of the slab was set at $320 \mathrm{~mm}$ as same as the thickness of the RC 
slab cut from a highway bridge. A disk-shaped void portion was set in the model to simulate a horizontal crack. The diameter of the void portion was $200 \mathrm{~mm}$. The depth of the void portion was set at $60 \mathrm{~mm}$ that was equivalent to the distance from the bottom surface of the slab to the tensile reinforcement. Solid elements defined by eight nodal points were adopted for the model. One side of an element had a length of approximately $10 \mathrm{~mm}$. The model was supported on four sides $80 \mathrm{~mm}$ x $80 \mathrm{~mm}$ each on corner of bottom surface (shaded part in Fig. 3). For the boundary condition, displacement at all nodal points in the shaded section (in $\mathrm{x}, \mathrm{y}$ and $\mathrm{z}$ directions) were fixed. An impact force was applied at a nodal point at the center of the bottom surface (shown in Fig. 3). The point of output was $50 \mathrm{~mm}$ away from the point of input.

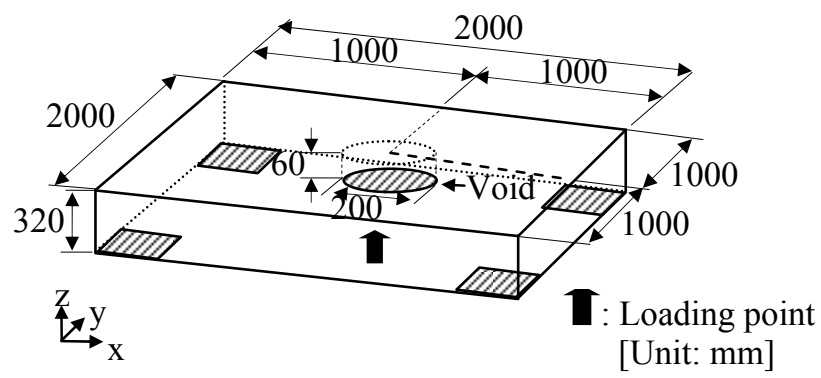

Fig. 3. Analysis model with void portion.

\subsection{Selection of elastic-wave input method}

It has been known that the characteristics of frequency of elastic-waves produced by tapping a steel ball against the slab surface are affected by the diameter of a steel ball ${ }^{2}$. In order to determine "I: elastic-wave input method", impact response analysis was conducted by simulating the tapping by steel balls with various diameters. The frequency spectra obtained by impact response analysis are shown in Fig. 4.

A steel ball was selected from among three steel balls that are capable of evaluating both models with and without void. Acceleration waveform was selected as output and maximum entropy method was adopted as the frequency analysis method.

The arrows show the theoretical peak frequency corresponding to the model thickness $f_{T}$. The broken lines indicate the theoretical peak frequency corresponding to the crack depth $f_{d}$. In the frequency spectrum obtained using a model without a void (shown in Fig. 4. a)), a peak is clearly identified nearly at the position of $f_{T}$ at all steel balls of diameters. It was considered that slab thickness could be estimated properly in cases where a steel ball of 6.4, 12.8 and $15.7 \mathrm{~mm}$ diameter was used for tapping the slab surface.

Discussed next is the frequency area near $f_{d}$ in the frequency spectrum obtained using a model with void (shown in Fig. 4. b)). Frequency peaked near $f_{d}$ in $6.4 \mathrm{~mm}$ cases except the cases where a steel ball of 12.8 or $15.7 \mathrm{~mm}$ diameter was used. It was concluded based on the above discussions that a steel ball of $6.4 \mathrm{~mm}$ diameter was the most appropriate for evaluating both slab thickness and void depth. 


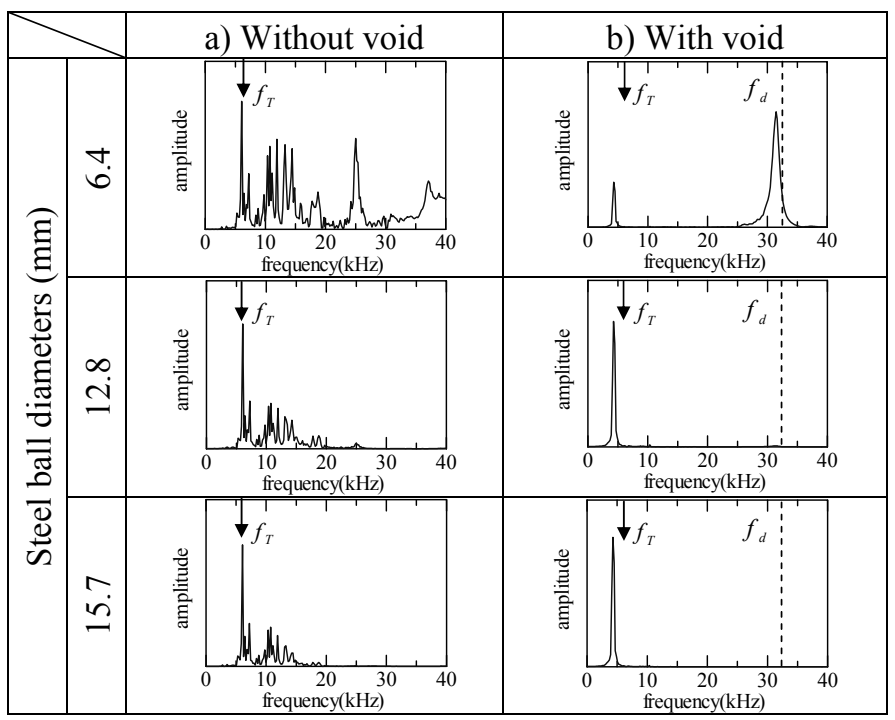

Fig. 4. Frequency spectra for different steel ball diameters.

\subsection{Selection of wave output positions}

In this study, optimal distance between impact point and output point of elastic-wave was determined by impact response analysis. An impact force was applied at a nodal point at the center of analysis model (shown in Fig. 3). Frequency spectra obtained by impact response analysis are shown in Fig. 5.

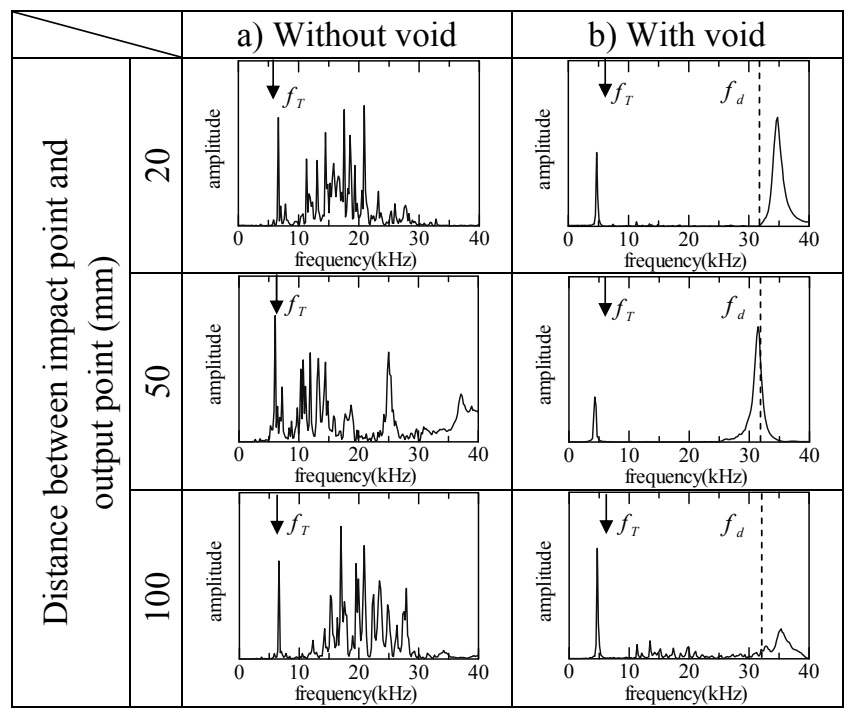

Fig. 5. Frequency spectra for different distance between impact point and output point. 
Points of output were set at 20,50 and $100 \mathrm{~mm}$ away from the input point in $\mathrm{x}$ direction. In these frequency spectra obtained using a model without a void, a peak clearly appeared nearly at the position of $f_{T}$ at all distance. In the cases of distance of 20 and $100 \mathrm{~mm}$, however, peaks also identified at other locations near $f_{T}$.

On the other hand, in these frequency spectra obtained using a model with void, a peak is clearly identified near $f_{d}$ at distance of 20 and $50 \mathrm{~mm}$. Therefore, it was concluded that distance between input point and output point of $50 \mathrm{~mm}$ was appropriate for evaluating both slab thickness and void depth.

\section{A Case Study of Measurement of a Reinforced Concrete Slab Cut from a Highway Bridge}

\subsection{Outline of measurement}

In order to verify the validity of "I: elastic-wave input method (tapping with a steel ball of $6.4 \mathrm{~mm}$ diameter)" and "II: elastic-wave detection method (distance between impact point and output point $50 \mathrm{~mm}$ )", measurements were performed for a RC slab cut from a highway bridge as a case study (shown in Fig. 6).

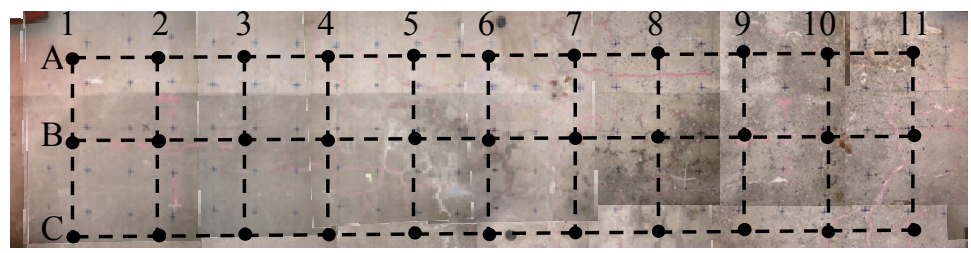

Fig. 6. Outline of measurement points by impact elastic-wave method.

\subsection{Results of measurement and discussions}

Examples of characteristic frequencies obtained at several measurement points by impact elastic-wave method are shown in Fig. 7. This figure shows that a peak frequency in the frequency spectrum was identical to $f_{T}$ at measurement point A2 and A5. It therefore seems that no horizontal cracks existed at the point A2 and A5.

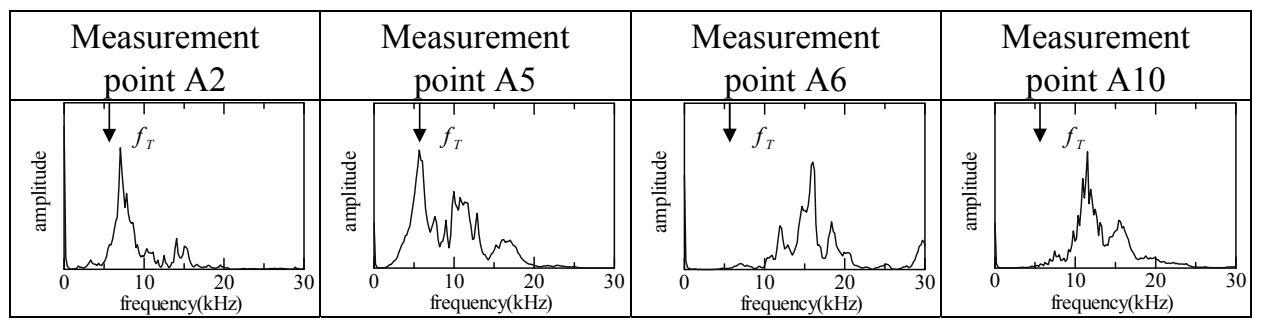

Fig. 7. Examples of frequency spectra obtained in measurement.

In contrast, a single peak appeared in the area of frequency higher than $f_{T}$ at 
measurement points A6 and A10. The peak frequency in the frequency spectrum was 16 $\mathrm{kHz}$ at measurement point $\mathrm{A} 6$ and $12 \mathrm{kHz}$ at point $\mathrm{A} 10$. Thus, there may be a possibility that horizontal cracks existed at depths of 125 and $167 \mathrm{~mm}$ from the bottom surface of the slab at measurement points A6 and A10, respectively (where $C_{p}$ was $4000 \mathrm{~m} / \mathrm{sec}$ ). In order to verify the validity of the results of estimation by non-destructive measurement, holes of $25 \mathrm{~mm}$ diameter were drilled at measurement points A5 and A6. After the holes were drilled, rod-shaped scanner was inserted into the holes to film the internal cracks. It was thus clarified that the results of estimation by the analysis-aided impact elastic-wave method were in good agreement with visual inspection results (shown in Fig. 8).

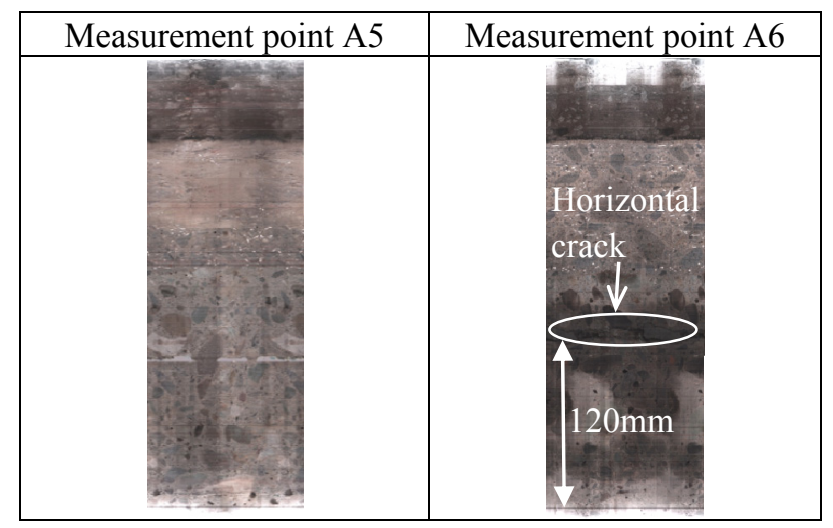

Fig. 8. Panoramic images by rod-shaped scanner.

\section{Conclusions}

The following conclusions were obtained in this study.

(1) As a better non-destructive method to detect horizontal cracks in RC slabs, a method was proposed for determining elastic wave input method and elastic wave detection method by impact response analysis before measurements by the impact elasticwave method.

(2) The proposed analysis-aided impact elastic-wave method was applied to a RC slab cut from an existing highway bridge as a case study. Obtained results by the method showed good agreement with the depths of horizontal cracks measured directly in boreholes.

\section{References}

1. T. Kamada, S. Uchida, K. Nakayama, H. Mae and T. Tamakoshi, Nondestructive Evaluation of Horizontal Cracks in RC Slabs by Impact Elastic-Wave Methods, in Proceedings of the 6th International Conference on Concrete under Severe Conditions, Environment \& Loading, Consec'10, Vol. 1 (2010. 6), pp.117-126.

2. T. Kamada, S. Uchida and K. Nakayama, Detecting Horizontal Cracks in RC Slabs with Asphalt Overlays Using Impact Elastic-Wave Methods, in Proceedings of the 7th International Conference on Fracture Mechanics of Concrete and Concrete Structures, FraMCoS-7, Vol. 2 (2010.5), pp.1116-1122. 